\title{
Ovarian Myxoid Liposarcoma
}

National Cancer Institute

\section{Source}

National Cancer Institute. Ovarian Myxoid Liposarcoma. NCI Thesaurus. Code C5235.

A liposarcoma that arises from the ovary and is composed of round to oval

mesenchymal cells, small signet ring lipoblasts, and a rich network of capillaries in a myxoid stroma. 